\title{
Atomic and Electronic Structure of Graphene Based on HRTEM and ab-initio Calculations
}

\author{
P. Plachinda,* S. Rouvimov,* G. Nandamuri,* R. Solanki*, K. Langworthy** \\ *Department of Physics, Portland State University, Portland, OR \\ **CAMCOR, University of Oregon, Eugene, OR
}

Graphene [1], probably the simplest of all graphitic forms, has recently become the focus of many research groups due to its unique physical properties such as a quantum Hall effect at room temperature, tunable band gap, an ambipolar electric field effect and high elasticity (see e.g. review paper of Ref. [2]). While its atomic structure seems to be simple (it constitutes of carbon atoms arranged in six-membered rings), the direct observation of single-layer graphene structure by High-Resolution Transmission Electron Microscopy (HRTEM) appeared to be challenging due to low contrast and, thus, requires special imaging conditions [3]. This paper demonstrates the effect of number of graphene layers and imaging conditions on HRTEM contrast based on multislice simulations as well as illustrates the electron density distribution in the film based on ab-initio calculations. Graphene films were grown on the Ni substrates [4] which were transferred to copper grids for electron microscopy analysis. FEI's Titan microscope (equipped with Cs-corrector) operated at $80 \mathrm{kV}$ was employed to view the graphene films at atomic resolution. Figure 1a shows high resolution electron microscope (HREM) image of single-, bi- and multilayer graphene films, whereas Figure 1b shows HREM image of a single-layer graphene region. Figure 1c shows enlarged region of Figure $1 \mathrm{~b}$ (marked by a red rectangle) and atomic sketch of graphene structure. As it has been shown earlier [3], the AB stacked bilayer regions show a qualitatively different pattern than the single-layer area, but care must be taken in using such images for identification because the singlelayer region may appear like a bilayer (and vice versa) at different defocus conditions. Figure 2 shows the effect of defocus on HRTEM contrast of a single and bi-layer graphene based on computer simulations. One can see that carbon atomic positions could be resolved in single- layer graphene films (Fig. 2, images of the top row) while it may be challenging in case of bi- (Fig. 2, images of the bottom row) and mulilayer graphene. Figure 3 show the electron density distributions in the graphene and graphite films based on ab-initio calculations. Valence electron density in graphene was computed using the ABINIT program complex [5]. The DFT theory [6] with Perdew-Burke-Ernzerhof GGA functional [7] has been employed for the computation. The Fig. 3 clearly reveals sp2 bonding within the honeycomb layer of graphene where bonds do not extend along the $\mathrm{z}$ axis, in contrast to bonding in graphite. Further work will be focused on examination of separate quantum states responsible for unusual electro-physical and mechanical properties of graphene.

\section{References}

[1] K. S. Novoselov et. al., Science, 306 (2004) 666.

[2] C. N. R. Rao et. al., Angew. Chem. Int. Ed. 2009, 48, 7752 - 7777 
[3] J. C. Meyer et.al., Nano Lett. 8 (2008), 3582-3586

[4] G. Nandamuri, S. Roumimov, and R. Solanki, (2010), in press

[5] Xavier Gonze, et. al., Z. Kristallogr. 220 (2005) 558-562

[6] D. Sholl, J. A. Steckel, Density Functional Theory: A Practical Introduction, Wiley, 2009

[7] J. P. Perdew, K. Burke, and M. Ernzerhof, Phys. Rev. Lett. 77 (1996) 3865

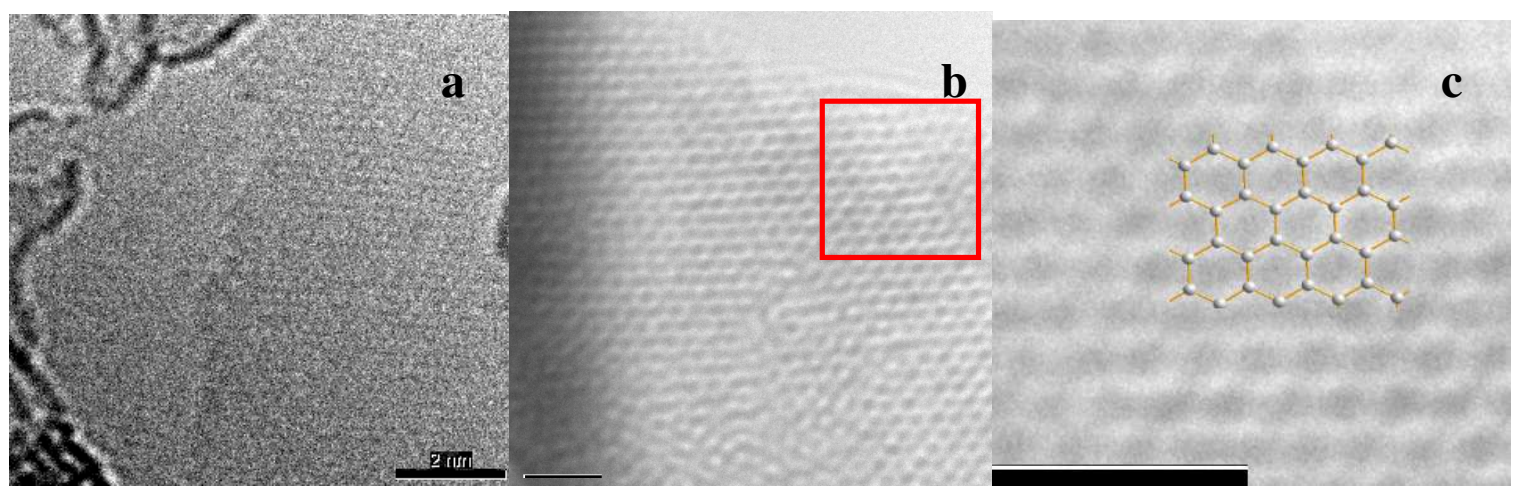

FIG. 1: High resolution transmission electron microscope (HRTEM) images of graphene sheets taken on FEI Titan on the edge of a film. The structures near the edge of the image (a) are adsorbates (possibly of iron oxide). HRTEM image (c) of a single-layer graphene is enlarged region of (b) outlined by a red rectangular. The bars of images (b) and (c) are $1 \mathrm{~nm}$.
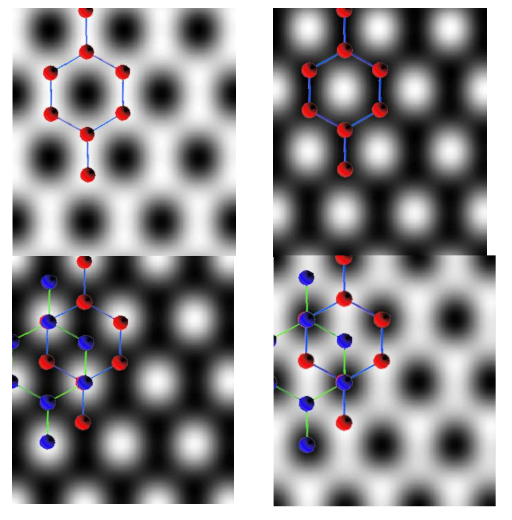

FIG. 3: The electron density distribution in the graphene (left) and graphite (right) based on ab-initio calculations.
FIG. 2: Computer simulations of HRTEM contrast of singleand bi-layer graphene (the first and second rows, respectively) at defoci of 0 and $-60 \mathrm{~nm}$ (the left and right columns, respectively). The atoms in the first and second graphene sublayes are shown in red and blue colors, respectively. Note: Contrast on the simulated images has been maximized and, therefore, looks much higher as compared to experimental images of Fig. 1.
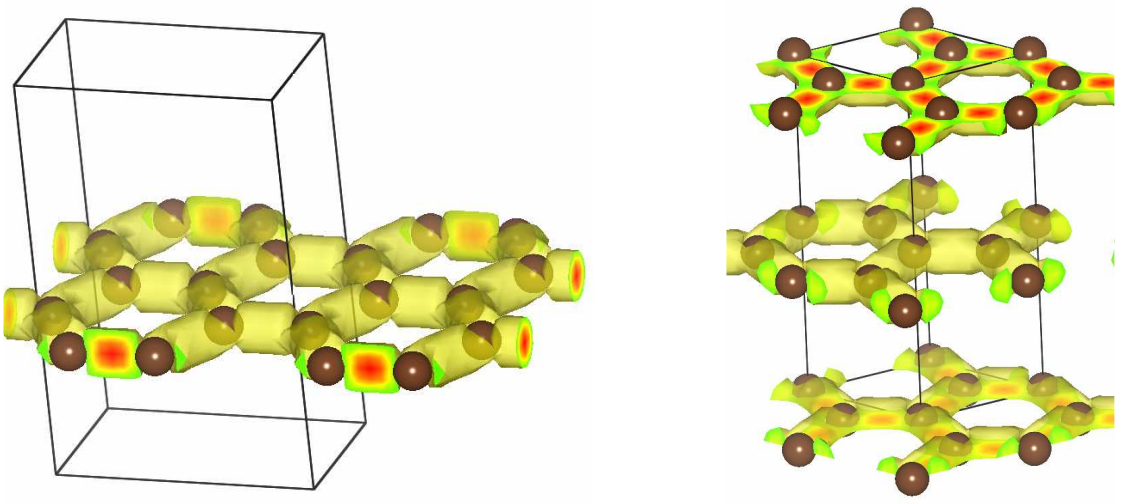